\title{
Nonlinear optics in the fractional quantum Hall regime
}

\section{Journal Article}

Author(s):

Knüppel, Patrick (i); Ravets, Sylvain; Kroner, Martin (1); Fält, Stefan; Wegscheider, Werner; Imamoglu, Atac

Publication date:

2019-08-01

Permanent link:

https://doi.org/10.3929/ethz-b-000352116

\section{Rights / license:}

In Copyright - Non-Commercial Use Permitted

Originally published in:

Nature 572(7767), https://doi.org/10.1038/s41586-019-1356-3

\section{Funding acknowledgement:}

671000 - Interacting polaritons in two-dimensional electron systems (EC) 


\title{
Nonlinear optics in the fractional quantum Hall regime
}

\author{
Patrick Knüppel*1, Sylvain Ravets*1,2, Martin Kroner ${ }^{1}$, Stefan Fält1,3, Werner Wegscheider ${ }^{3}$, \\ and Atac Imamoglu ${ }^{1}$ \\ ${ }^{1}$ Institute of Quantum Electroncis, ETH Zürich, CH-8093 Zürich, Switzerland \\ ${ }^{2}$ CNRS Centre for Nanoscience and Nanotechnology, Université Paris-Sud, Université Paris-Saclay, \\ 91120 Palaiseau, France \\ ${ }^{3}$ Solid State Physics Laboratory, ETH Zürich, CH-8093 Zürich, Switzerland \\ ${ }^{*}$ These authors contributed equally to this work.
}

Engineering strong interactions between optical photons is a great challenge for quantum science. Envisioned applications range from the realization of photonic gates for quantum information processing ${ }^{1}$ to synthesis of photonic quantum materials for investigation of stronglycorrelated driven-dissipative systems. ${ }^{2}$ Polaritonics, based on the strong coupling of photons to atomic or electronic excitations in an optical resonator, has emerged as a promising approach to implement those tasks. ${ }^{3}$ Recent experiments demonstrated the onset of quantum correlations in the exciton-polariton system, ${ }^{4,5}$ showing that strong polariton blockade ${ }^{6}$ could be achieved if interactions were an order of magnitude stronger. Here, we report time resolved four-wave mixing experiments on a two-dimensional electron system embedded in an optical cavity, ${ }^{7}$ demonstrating that polariton-polariton interactions are strongly enhanced when the electrons are initially in a fractional quantum Hall state. Our experiments indicate that in addition to strong correlations in the electronic ground state, exciton-electron interactions leading to the formation of polaron polaritons ${ }^{8-11}$ play a key role in enhancing the nonlinear optical response. Besides potential applications in realization of strongly interacting photonic systems, our findings suggest that nonlinear optical measurements could provide information about fractional quantum Hall states that is not accessible in linear optical response.

Polaritons have recently attracted considerable interest, motivated by the fact that their interactions can be engineered almost at will through the tunability of their matter component. For example, strongly interacting Rydberg polaritons have recently been obtained using the nonlinear behavior of Rydberg excitations in an ensemble of atoms, which led to the demonstration of Rydberg polariton blockade ${ }^{12}$ where the presence of a single polariton in a well-delimited region of space prevents the resonant injection of other polaritons. In parallel, efforts are being made to realize polariton blockade in condensed matter systems that hold great potential for realizing compact and integrated synthetic quantum materials. ${ }^{3}$ Exciton polaritons in semiconductor materials are part light part matter particles that arise from the strong coupling of a quantum well (QW) exciton and a cavity photon. ${ }^{13}$ These photonic particles inherit a nonlinear behavior from exciton-exciton interactions. ${ }^{2,13}$ For efficient blockade to be obtained, the polariton interaction energy $U_{\mathrm{p}}$ needs to be greater than the inverse lifetime $\gamma$ of the polaritons. ${ }^{6}$ Recent state-of-the art experiments based on photon correlation measurements in semi-integrated microcavities attained optimized values of the ratio $U_{\mathrm{p}} / \gamma \simeq 0.1$ in a photonic dot with about $3 \mu \mathrm{m}^{2}$ area. ${ }^{4,5}$ These experiments represent the culmination of decade long technological developments aimed at increasing $U_{\mathrm{p}} / \gamma$ through reducing the photonic mode area ${ }^{4,5,14}$ as well as increasing the lifetime. ${ }^{15}$ Recently, several possibilities have been explored for enhancing $U_{\mathrm{p}}$ through an increase of exciton-exciton interactions $U$, focusing either on biexciton Feshbach resonance ${ }^{16}$ or on excitons with a permanent dipole moment. ${ }^{17-19}$ The experiments we report here reveal a hitherto unexplored mechanism for optical nonlinearity emerging for polaritonic excitations out of a two dimensional electron system (2DES) in the fractional quantum Hall (FQHE) regime. Using time resolved four-wave mixing (FWM) experiments (see Methods), we find that polaron-polaron interactions $U$ are enhanced by more than an order of magnitude around the fractional state at filling factor $\nu=2 / 5$ as compared to other neighboring compressible states. A comparison of $U$ for $\nu=2 / 5$ shows a factor of $4.6 \pm 0.9$ enhancement with respect to an undoped QW. Moreover, we find that the lower polariton linewidth $\gamma$ in our sample is similar to the values reported in recent experiments demonstrating modest quantum correlations, ${ }^{4,5}$ indicating that upon spatial confinement polariton blockade regime could be reached. The interplay between photonic excitations and a 2DES is an exciting field ${ }^{20-24}$ with open problems, among others, concerning the relation between transport and optics ${ }^{25,26}$ and the description of exciton-electron interactions in a magnetic field. ${ }^{10}$

We study a semiconductor heterostructure that features, at the center of an optical microcavity, a GaAs QW containing an electron system of density $n_{e}=3 \times 10^{10} \mathrm{~cm}^{-2}$ (see Methods). In the presence of a 2DES, 
electron-exciton interactions modify the excitation spectrum and pioneering studies showed the existence of the strong coupling regime. ${ }^{27,28}$ A consistent description of the new excitonic excitations that emerge in the presence of a 2DES was first provided by R. Suris, ${ }^{29}$ and were later termed exciton-polarons. ${ }^{8-10}$ Strong coupling to an optical mode in turn results in the formation of polaron-polaritons as the elementary excitations of the 2DES-cavity system. ${ }^{9,11}$ Under an external magnetic field $B$ orthogonal to the 2DES surface, discrete Landau levels $\mathrm{LL} n_{e}\left(\mathrm{LL} n_{\mathrm{hh}}\right)$ form out of the conduction (heavy-hole valence) band. We focus, in this article, on resonant optical excitations associated with the lowest Landau level LL0. As we increase $B$, the filling factor $\nu$ of LL0 decreases, allowing us to reach the fractional quantum Hall regime. We address transitions to the $|\uparrow\rangle(|\downarrow\rangle)$ LL0 subband using $\sigma^{-}\left(\sigma^{+}\right)$circularly-polarized light.

We first characterize our sample using optical spectroscopy in the low-power (linear) regime. The sample is mounted inside a dilution refrigerator with fibered optical access, as shown in Fig. 1a and b. We record reflectivity spectra for several values of $\nu$ using circularly-polarized light from a broadband light source. Fig. 1c plots an overview of the polaron-polariton lines for our system, obtained by calculating the difference between the spectra measured using $\sigma^{-}$(red) and $\sigma^{+}$(blue) polarized light. We observe generic strong dispersion of the polariton energies with magnetic field around integer and fractional values of $\nu$. This striking behavior of the linear optical spectrum stems from strong modification of electron-exciton interactions in and around gapped quantum Hall states, ${ }^{11}$ which in turn leads to a $\nu$-dependent modification of the cavity-polaron coupling strength.

Figure 2a shows the principle of the time-resolved interferometer we developed, inspired by traditional FWM techniques (see Methods), to characterize our sample in the nonlinear regime. We use a pulsed Ti:Sapphire laser with a $T_{\text {pulse }}=4 \mathrm{ps}$ pulse duration, a $76 \mathrm{MHz}$ repetition rate and center frequency tuned to the $\sigma^{-}$polarization lower polariton resonance $\mathrm{LP}_{\sigma^{-}}$. We split the laser into two paths and introduce a variable time delay $\tau$ between the two pulses. For optical excitation, we recombine both beams onto a beam splitter and couple the linearlypolarized light into an optical fiber routed to the sample. The excitation light is then focused onto the sample surface using a low NA objective. The total field incident on the sample is given by $E(t, \tau)=E_{1}(t)+E_{2}(t, \tau)$, where the average intensities of the two beams are chosen to be equal. For detection, we collect the generated resonance fluorescence using the same fiber as the one used for excitation, and we filter out the laser background light by detecting along the cross-polarized axis. The collected light is finally sent onto an avalanche photodiode (APD) for detection. Modeling our system as a third order nonlinear medium, we can expand the total intensity reaching the photodetector $I_{\text {det }}$ as:

$$
I_{\mathrm{det}}(t, \tau) \propto \epsilon_{0}\left|P_{1}^{(1)}(t)+P_{2}^{(1)}(t, \tau)+P^{(3)}(t, \tau)\right|^{2},
$$

where the linear $P_{1,2}^{(1)}(t)$ (nonlinear $P^{(3)}(t)$ ) polarizations are the inverse Fourier transforms of $P_{1,2}^{(1)}(\omega)\left(P^{(3)}(\omega)\right.$ ). To isolate weaker nonlinear terms $\propto P_{i}^{(1) *} P^{(3)}$ from the dominant linear contributions $\propto P_{i}^{(1) *} P_{j}^{(1)}(i, j=$ $1,2)$, we modulate the field amplitude $E_{1}(t)$ sinusoidally at frequency $\omega_{\mathrm{m}}$. By calculating $\mathcal{I}(\omega, \tau)$, the Fourier transform of $I_{\text {det }}(t, \tau)$, we can separate different terms: the (mostly) linear term $\mathcal{I}\left(\omega_{\mathrm{m}}, \tau\right)$ and the nonlinear term $\mathcal{I}\left(3 \omega_{\mathrm{m}}, \tau\right)$ (see Methods). In the following, we use these two terms to quantify the nonlinearity of the system.

We now focus on pump-probe measurements around $\nu=2 / 5(B=3.15 \mathrm{~T})$. We observe, in Fig. $2 \mathrm{~b}$, that $\mathcal{I}\left(\omega_{\mathrm{m}}, \tau\right)$ features a fast oscillation modulated by an exponential envelope. This is the expected waveform since $\mathcal{I}\left(\omega_{\mathrm{m}}, \tau\right)$ is, to lowest order, the autocorrelation signal of the resonance fluorescence emitted by the sample: the carrier frequency of the fast oscillation corresponds to the (undersampled) optical frequency and the characteristic decay time is the polariton coherence time $T_{\mathrm{LP}}=24 \pm 1 \mathrm{ps}$ (dashed black line). The nonlinear contribution $\mathcal{I}\left(3 \omega_{\mathrm{m}}, \tau\right)$, depicted in Fig 2c, also exhibits fast oscillations but its envelope has a more complex structure as a consequence of the interplay between several interfering nonlinear terms, with characteristic decay times $T_{\mathrm{LP}}$ and $T_{\mathrm{LP}} / 3$ that compensate at short delays. Figure $2 \mathrm{~d}$ shows a logarithmic plot of the integrals $\left\langle\mathcal{I}\left(\omega_{\mathrm{m}}, \tau\right)\right\rangle_{\tau}=\int \mathcal{I}\left(\omega_{\mathrm{m}}, \tau\right) d \tau$ and $\left\langle\mathcal{I}\left(3 \omega_{\mathrm{m}}, \tau\right)\right\rangle_{\tau}=\int \mathcal{I}\left(3 \omega_{\mathrm{m}}, \tau\right) d \tau$ as a function of the average incident power. We observe that the former exhibits a power law with exponent $1.3 \pm 0.3$, which is consistent with the expected linear behavior. By contrast, $\left\langle\mathcal{I}\left(3 \omega_{\mathrm{m}}, \tau\right)\right\rangle_{\tau}$ shows a power law with exponent $2.2 \pm 0.3$ that is consistent with the anticipated dependence of third-order nonlinear response, validating that $\left\langle\mathcal{I}\left(3 \omega_{\mathrm{m}}, \tau\right)\right\rangle_{\tau}$ is indeed a good measure of the nonlinearity. The observed deviation of the power law exponents from the expected values 1.0 and 2.0 is most likely due to systematic errors on the input power calibration. We emphasize that the measured nonlinearity occurs on timescales that are comparable to the polariton lifetime, which demonstrates that our method allows us to access (fast) polariton-polariton interactions. We also note that the nonlinear response saturates at high optical powers (Fig. 2c). The saturation behaviour of $\left\langle\mathcal{I}\left(3 \omega_{\mathrm{m}}, \tau\right)\right\rangle_{\tau}$ at high optical powers may be attributed to the saturation of the $\mathrm{LP}_{\sigma^{-}}$red shift induced by a change in $\nu$ (Fig. 1c). Saturation could also be a consequence of (slow) light-induced modifications of $n_{e}$, which may start to play a role at the highest powers investigated. ${ }^{11}$

We now analyze the evolution of $\left\langle\mathcal{I}\left(\omega_{\mathrm{m}}, \tau\right)\right\rangle_{\tau}$ and $\left\langle\mathcal{I}\left(3 \omega_{\mathrm{m}}, \tau\right)\right\rangle_{\tau}$ as a function of $\nu$ by measuring $I(t, \tau)$ for different values of $B$. The data consists of three sets centered around filling factors $1,2 / 3$, and $2 / 5$ that exhibit 
clear signatures of optical coupling to quantum Hall states, as demonstrated in Fig. 1c. For each dataset, we tune $B$ to access neighboring filling factors while keeping the cavity energy constant and adjusting the laser frequency to resonantly excite $\mathrm{LP}_{\sigma^{-}}$. The main result of this letter is the remarkable $\nu$-dependence of the nonlinear signal $\mathcal{I}\left(3 \omega_{\mathrm{m}}, \tau\right)$ shown in Fig. 3a. We observe a strong increase of the nonlinearity at fractional filling factors $\nu=2 / 3(B \simeq 1.95 \mathrm{~T})$ and $\nu=2 / 5(B \simeq 3.15 \mathrm{~T})$, as compared to neighboring filling factors. Away from these states, e.g. for $B=3.5 \mathrm{~T}$, the nonlinearity becomes weaker and eventually comparable to the noise level of our apparatus. This gives clear evidence that polariton-polariton interactions are enhanced around the fractional quantum Hall states $\nu=2 / 3$ and $\nu=2 / 5$. In stark contrast, we observe that $\mathcal{I}\left(3 \omega_{\mathrm{m}}, \tau\right)$ is only marginally modified around the integer filling factor $\nu=1$. We summarize our results in Fig. 3c, where we provide values of the enhancement of polariton-polariton interactions close to fractional filling factors, obtained by calculating the ratio of the areas $R_{\mathrm{a}}=\left\langle\mathcal{I}\left(3 \omega_{\mathrm{m}}, \tau\right)\right\rangle_{\tau} /\left\langle\mathcal{I}\left(\omega_{\mathrm{m}}, \tau\right)\right\rangle_{\tau}$ and the ratio of the signal peak-to-peak values $R_{\mathrm{pp}} \cdot{ }^{30}$ At $\nu=2 / 5$, both measures show a significant enhancement of the interaction, of the order of $10^{1}$, as compared to neighboring filling factors.

In order to quantify $U$ we solve the driven-dissipative Gross-Pitaevskii equation incorporating a Kerrnonlinearity. We obtain $U=57_{-7(40)}^{+7(80)} \mu \mathrm{eV} \mu \mathrm{m}^{2}$, where the first uncertainty is statistical (s.d.) and the second (in parenthesis), is our best estimate of systematic errors stemming from our estimation of the polariton number and mode area (see Methods). Although the absolute measurement of $U$ is susceptible to large systematic errors, the relative variation of $U$ is only susceptible to statistical errors. We validate our measurement by repeating the same procedure on a different sample featuring a neutral QW $\left(n_{e}=0\right)$. We obtain a value of $12.5_{-2(9)}^{+2(18)} \mu \mathrm{eV} \mu \mathrm{m}^{2}$ for the exciton-exciton interaction strength, which is compatible with values reported elsewhere. ${ }^{4,5,14,31-33}$ Although our model reproduces the delay-time-dependence of our measurements for the neutral QW, the observed response close to fractional filling factors is not fully captured by this description in terms of a Kerr nonlinearity (see Methods). This remark notwithstanding, we find that interactions are enhanced by a factor $4.6 \pm 0.9$ in the 2DES sample as compared to the neutral QW sample. We emphasize that the measured $\mathrm{LP}_{\sigma^{-}}$linewidth at $\nu=2 / 5, \gamma \simeq 67 \pm 5 \mu \mathrm{eV}$, is comparable to linewidths used in recent state of the art experiments. $^{4,5}$ Morever, another striking feature coincides with the enhancement of the nonlinearity (see Figure 3b): for $\nu=2 / 3$ and $\nu=2 / 5$ we find that $T_{\mathrm{LP}} \propto \gamma^{-1}$ is prolonged by a factor two to three as we increase the incident optical power (see Methods). Although the origin of this power dependent enhancement of $T_{\mathrm{LP}}$ remains unclear, this observation shows that the presence of a 2DES at finite fields has no adverse effect on $\gamma$.

We speculate that the enhanced optical nonlinearity we observe for polaritons generated by $\sigma^{-}$excitation is a consequence of the fragility of FQHE states against changes in $\nu$. Due to the absence of electron-hole symmetry at low $B$ fields, optical excitation of an electron to the spin-up LL0 modifies the effective filling factor by creating quasiparticles. The size of the incompressbility gap should determine the number of extra electrons that can be excited (by photons) before the electronic system becomes compressible. Optical modification of the electronic ground-state in turn modifies the ability of the electrons to dynamically screen the excitons, resulting in a conditional shift of the polaron-polariton resonance that underlies the nonlinear response. In contrast, for IQHE state $\nu=1$, we do not observe a significant enhancement of interactions because the electronic state is robust against small deviations in the filling factor, which in turn leads to a small modification of the photonic resonances.

Further experimental evidence supporting this tentative explanation is provided by exciting the $\mathrm{LP}_{\sigma^{+}}$reso- $^{-}$ nance at $\nu=2 / 5$, where we did not observe any enhancement of the nonlinearity. Since $\sigma^{+}$excitation generates an electron in the spin-down LL0, it does not lead to a direct modification of $\nu$; the absence of an enhancement of nonlinearity for $\sigma^{+}$excitation is therefore consistent with our explanation. We repeated the experiment around the $\nu=1 / 3$ state $(B=3.9 \mathrm{~T})$, where we also did not observe an enhanced nonlinearity. We argue that this is due to the suppression of $\sigma^{-}$polarized polaron formation due to perfect spin polarization of the 2DES at $\nu \leq 1 / 3$, leaving the polariton mode mostly photonic. ${ }^{11}$ The large incompressibility gap of $\nu=1 / 3$ may also suppress the nonlinearity. Last but not least, we measured enhanced nonlinearity also on a second sample with higher electron density $\left(n_{e}=1.4 \times 10^{11} \mathrm{~cm}^{-2}\right)$ for the (spin-polarized) $\nu=2 / 3$ state at $B \simeq 8.5 \mathrm{~T}($ see Methods).

Strong enhancement of polariton-polariton interactions around FQHE states opens up new perspectives for the study of strongly correlated electron as well as for photonic systems. Embedding our sample in a zero-dimensional cavity with a mode area of $A=1 \mu \mathrm{m}^{2}$ would yield $U /(A \gamma)=0.85$, which indicates that polariton blockade regime is within reach. Understanding the physical mechanism for enhanced nonlinear response and prolonged polariton coherence times for FQHE states constitutes a very interesting open problem. Our experiments show that despite their qualitatively similar linear optical response, fractional and integer QHE states show strikingly different nonlinear optical signatures: this suggests that nonlinear spectroscopy could reveal signatures of strongly correlated electronic systems that are not accessible by linear optical or transport measurements. 


\section{References}

[1] O'Brien, J. L., Furusawa, A. \& Vučković, J. Photonic quantum technologies. Nature Photonics 3, 687-695 (2009). URL http://www. nature.com/articles/nphoton.2009.229.

[2] Carusotto, I. \& Ciuti, C. Quantum fluids of light. Reviews of Modern Physics 85, 299-366 (2013). URL https://link.aps.org/doi/10.1103/RevModPhys.85.299.

[3] Sanvitto, D. \& Kéna-Cohen, S. The road towards polaritonic devices. Nature Materials 15, 1061-1073 (2016). URL https://www. nature.com/articles/nmat4668.

[4] Muñoz-Matutano, G. et al. Emergence of quantum correlations from interacting fibre-cavity polaritons. Nature Materials 18, 213 (2019). URL https://www nature.com/articles/s41563-019-0281-z.

[5] Delteil, A. et al. Towards polariton blockade of confined exciton-polaritons. Nature Materials 18, 219 (2019). URL https://www.nature.com/articles/s41563-019-0282-y.

[6] Verger, A., Ciuti, C. \& Carusotto, I. Polariton quantum blockade in a photonic dot. Physical Review B 73, 193306 (2006). URL https://link.aps.org/doi/10.1103/PhysRevB.73.193306.

[7] Smolka, S. et al. Cavity quantum electrodynamics with many-body states of a two-dimensional electron gas. Science 346, 332-335 (2014). URL http://science. sciencemag. org/content/346/6207/332.

[8] Schmidt, R., Enss, T., Pietilä, V. \& Demler, E. Fermi polarons in two dimensions. Physical Review A 85 (2012). URL https://link.aps.org/doi/10.1103/PhysRevA.85.021602.

[9] Sidler, M. et al. Fermi polaron-polaritons in charge-tunable atomically thin semiconductors. Nature Physics 13, 255-261 (2017). URL https://www.nature.com/articles/nphys3949.

[10] Efimkin, D. K. \& MacDonald, A. H. Exciton-polarons in doped semiconductors in a strong magnetic field. Physical Review B 97 (2018). URL https://link.aps.org/doi/10.1103/PhysRevB.97.235432.

[11] Ravets, S. et al. Polaron Polaritons in the Integer and Fractional Quantum Hall Regimes. Physical Review Letters 120, 057401 (2018). URL https://link.aps.org/doi/10.1103/PhysRevLett.120.057401.

[12] Jia, N. et al. A strongly interacting polaritonic quantum dot. Nature Physics 14, 550-554 (2018). URL https://www . nature. com/articles/s41567-018-0071-6.

[13] Deng, H., Haug, H. \& Yamamoto, Y. Exciton-polariton Bose-Einstein condensation. Reviews of Modern Physics 82, 1489-1537 (2010). URL https://link.aps.org/doi/10.1103/RevModPhys.82.1489.

[14] Ferrier, L. et al. Interactions in Confined Polariton Condensates. Physical Review Letters 106 (2011). URL https://link.aps.org/doi/10.1103/PhysRevLett.106.126401.

[15] Sun, Y. et al. Bose-Einstein Condensation of Long-Lifetime Polaritons in Thermal Equilibrium. Physical Review Letters 118, 016602 (2017). URL https://link.aps.org/doi/10.1103/PhysRevLett.118.016602.

[16] Takemura, N., Trebaol, S., Wouters, M., Portella-Oberli, M. T. \& Deveaud, B. Polaritonic Feshbach resonance. Nature Physics 10, 500-504 (2014). URL https://www . nature.com/articles/nphys2999.

[17] Cristofolini, P. et al. Coupling Quantum Tunneling with Cavity Photons. Science 1219010 (2012). URL http://science.sciencemag.org/content/early/2012/04/05/science.1219010.

[18] Rosenberg, I. et al. Strongly interacting dipolar-polaritons. Science Advances 4, eaat8880 (2018). URL http://advances.sciencemag.org/content/4/10/eaat8880.

[19] Togan, E., Lim, H.-T., Faelt, S., Wegscheider, W. \& Imamoglu, A. Enhanced Interactions between Dipolar Polaritons. Physical Review Letters 121 (2018). URL https://link.aps.org/doi/10.1103/ PhysRevLett.121.227402.

[20] Kukushkin, I. V., v. Klitzing, K. \& Eberl, K. Spin Polarization of Composite Fermions: Measurements of the Fermi Energy. Physical Review Letters 82, 3665-3668 (1999). URL https://link.aps.org/doi/10. 1103/PhysRevLett.82.3665.

[21] Byszewski, M. et al. Optical probing of composite fermions in a two-dimensional electron gas. Nature Physics 2, 239-243 (2006). URL https://www.nature.com/articles/nphys273. 
[22] Groshaus, J. G. et al. Absorption in the Fractional Quantum Hall Regime: Trion Dichroism and Spin Polarization. Physical Review Letters 98, 156803 (2007). URL https://link.aps.org/doi/10.1103/ PhysRevLett.98.156803.

[23] Bar-Joseph, I. Trions in GaAs quantum wells. Semiconductor Science and Technology 20, R29 (2005). URL http://stacks.iop.org/0268-1242/20/i=6/a=R01.

[24] Hayakawa, J., Muraki, K. \& Yusa, G. Real-space imaging of fractional quantum Hall liquids. Nature Nanotechnology 8, 31-35 (2013). URL https://www.nature.com/articles/nnano.2012.209.

[25] Bartolo, N. \& Ciuti, C. Vacuum-dressed cavity magnetotransport of a two-dimensional electron gas. Physical Review B 98, 205301 (2018). URL https://link.aps.org/doi/10.1103/PhysRevB.98.205301.

[26] Paravicini-Bagliani, G. L. et al. Magneto-transport controlled by Landau polariton states. Nature Physics 15, 186 (2019). URL https://www.nature.com/articles/s41567-018-0346-y.

[27] Rapaport, R. et al. Negatively Charged Quantum Well Polaritons in a GaAs/AlAs Microcavity: An Analog of Atoms in a Cavity. Physical Review Letters 84, 1607-1610 (2000). URL https://link.aps.org/doi/ 10.1103/PhysRevLett.84.1607.

[28] Rapaport, R., Cohen, E., Ron, A., Linder, E. \& Pfeiffer, L. N. Negatively charged polaritons in a semiconductor microcavity. Physical Review B 63, 235310 (2001). URL https://link.aps.org/doi/10.1103/ PhysRevB.63.235310.

[29] Suris, R. A. Correlation Between Trion and Hole in Fermi Distribution in Process of Trion Photo-Excitation in Doped QWs. In Ossau, W. J. \& Suris, R. (eds.) Optical Properties of $2 D$ Systems with Interacting Electrons, NATO Science Series, 111-124 (Springer Netherlands, 2003).

[30] $R_{\mathrm{pp}}=\left(\max \left(\mathcal{I}\left(3 \omega_{\mathrm{m}}, \tau\right)\right)-\min \left(\mathcal{I}\left(3 \omega_{\mathrm{m}}, \tau\right)\right)\right) /\left(\max \left(\mathcal{I}\left(\omega_{\mathrm{m}}, \tau\right)\right)-\min \left(\mathcal{I}\left(\omega_{\mathrm{m}}, \tau\right)\right)\right)$.

[31] Rodriguez, S. R. K. et al. Interaction-induced hopping phase in driven-dissipative coupled photonic microcavities. Nature Communications 7, 11887 (2016). URL https://www.nature.com/articles/ ncomms11887.

[32] Brichkin, A. S. et al. Effect of Coulomb interaction on exciton-polariton condensates in GaAs pillar microcavities. Physical Review B 84 (2011). URL https://link.aps.org/doi/10.1103/PhysRevB.84. 195301.

[33] Walker, P. et al. Dark Solitons in High Velocity Waveguide Polariton Fluids. Physical Review Letters 119, 097403 (2017). URL https://link.aps.org/doi/10.1103/PhysRevLett.119.097403.

\section{Acknowledgements}

The Authors acknowledge fruitful discussions with Jacqueline Bloch, Antoine Browaeys, Thibault Chervy, Ovidiu Cotlet, Aymeric Delteil, Tobias Grass, Mohammad Hafezi, Emre Togan, Sina Zeytinoglu and Oded Zilberberg. We thank Mirko Lupatini for providing us with the neutral QW reference sample. This work was supported by the Swiss National Science Foundation (NCCR Quantum Science and Technology), an ETH Fellowship (S.R.). This project has received funding from the European Research Council under the Grant Agreement No 671000.

\section{Author contributions}

P.K. and S.R. contributed equally to this work. P.K. and S.R. performed and analyzed the measurements, S.F. and W.W. grew the sample. S.R., M.K. and A.I. supervised the work. P.K., S.R., M.K. and A.I. wrote the manuscript.

\section{Author information}

Rights and permissions. Reprints and permissions information is available at www.nature.com/reprints.

Competing interests. The authors declare no competing interests.

Correspondence and requests for materials should be addressed to A.I. (imamoglu@phys.ethz.ch) and S.R. (sylvain.ravets@u-psud.fr). 
a
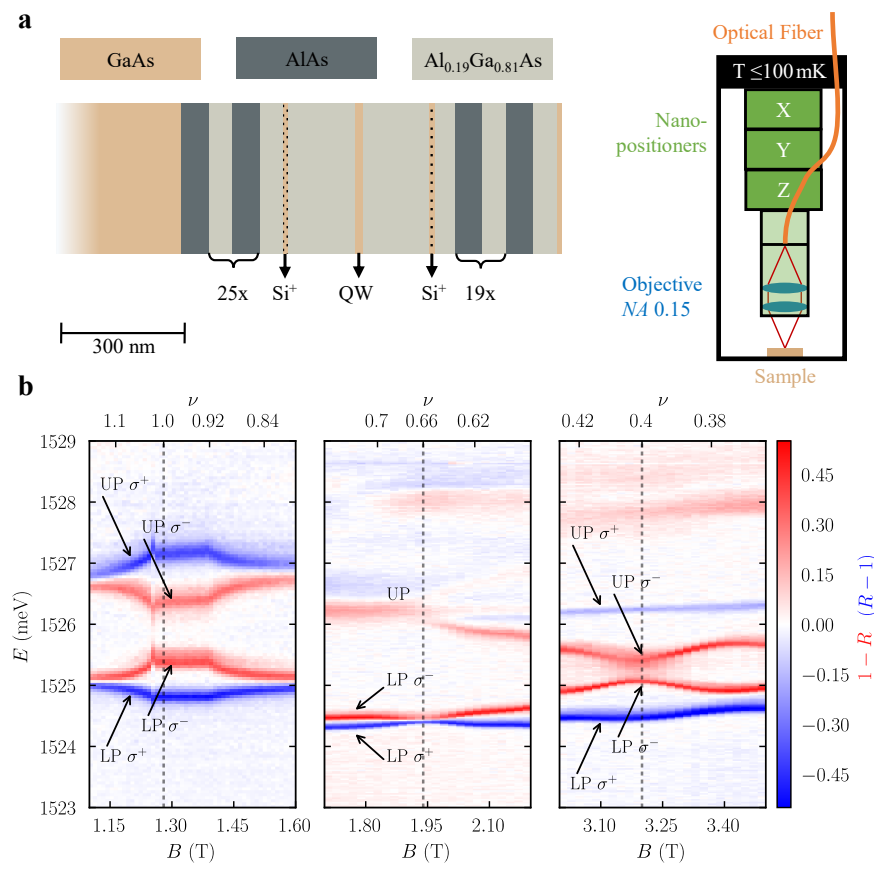

Figure 1: Quantum Hall polaritons. a, Sample structure and b, experimental setup for magneto-optical measurements at $\mathrm{mK}$ temperatures. c, White light reflectivity spectra recorded around filling factors $\nu=1$, $2 / 3$ and $2 / 5$. The plots show the difference between two spectra obtained separately using $\sigma^{-}$and $\sigma^{+}$polarized light.

\section{Methods}

Sample structure. Our sample structure, detailed in, ${ }^{11}$ features a $20 \mathrm{~nm}$ modulation doped gallium arsenide (GaAs) QW located at the center of a $2 \lambda \mathrm{Al}_{0.19} \mathrm{Ga}_{0.81} \mathrm{As}$ microcavity. The lower (upper) distributed Bragg reflectors consist of 25 (19) pairs of $\mathrm{AlAs} / \mathrm{Al}_{0.20} \mathrm{Ga}_{0.80} \mathrm{As}$ layers. The cavity quality factor, measured by white light reflectivity, is $Q \simeq(5.5 \pm 0.1) \times 10^{3}$. The 2 DES shows an electron density $n_{e} \simeq 0.33 \times 10^{11} \mathrm{~cm}^{-2}$ and a mobility $\mu \simeq 1.6 \times 10^{6} \mathrm{~cm}^{2} \mathrm{~V}^{-1} \mathrm{~s}^{-1}$, as measured by magneto-transport. Note that with these parameters, we conveniently access various integer and fractional quantum Hall states for relatively low magnetic fields $B \leq 5 \mathrm{~T}$. A thickness gradient across the sample allows us to tune the cavity energy by probing different positions on the sample.

Optical characterization. We perform an initial characterization of our sample by polarization-resolved white light reflectivity as a function of $B$. We couple light from a broadband light emitting diode into an optical fiber and shine a few nanowatts of optical power onto the sample placed inside a dilution refrigerator with a base temperature of $30 \mathrm{mK}$ (see Fig. 1b). Monitoring the polariton spectrum around $\nu=2 / 5$ while increasing the temperature suggests that the electron temperature is lower than $200 \mathrm{mK}$. The light is focused on the sample surface using a low numerical aperture lens $(N A=0.15)$ in confocal configuration. Reflected light is then collected by the same fiber and analyzed in the detection path (green line in Extended Data Fig. 1). The collected light is finally sent to a spectrometer equipped with a nitrogen-cooled CCD. Spectra $s(\omega)$ are recorded for different values of $B$. Concurrently, we determine the background reflection spectrum $r(\omega)$ of the incident light by monitoring $s(\omega)$ away from polaritonic resonances. Tuning the latter, by ramping the magentic field allows us to determine $r(\omega)$ for all $\omega$. We then calculate $1-s(\omega) / r(\omega)$, to obtain the background corrected spectra shown in Fig. 1b of the main text. Importantly, we are also able, based on the resonances observed in the white light reflectivity spectrum, to optimize carefully the polarization of the input light field to be either right-hand circularly polarized $\left(\sigma^{+}\right)$or left-hand circularly polarized $\left(\sigma^{-}\right)$.

Time-resolved four-wave mixing. One standard method for evaluating interactions between excitonpolaritons in $2 \mathrm{D}$ uses a resonant continuous wave excitation laser to monitor the blue-shift experienced by the lower polariton line due to the (Kerr-like) nonlinearity as the polariton population increases. In these experiments, however, one cannot differentiate between the contribution due to fast $(\sim 10$ ps $)$ polariton-polariton interactions, and other unwanted contributions due to the slow ( $>100 \mathrm{ps}$ ) buildup of an excitonic reservoir. ${ }^{34}$ This issue is critical in the context of quantum Hall polaritons since the 2DES electron density is particularly 

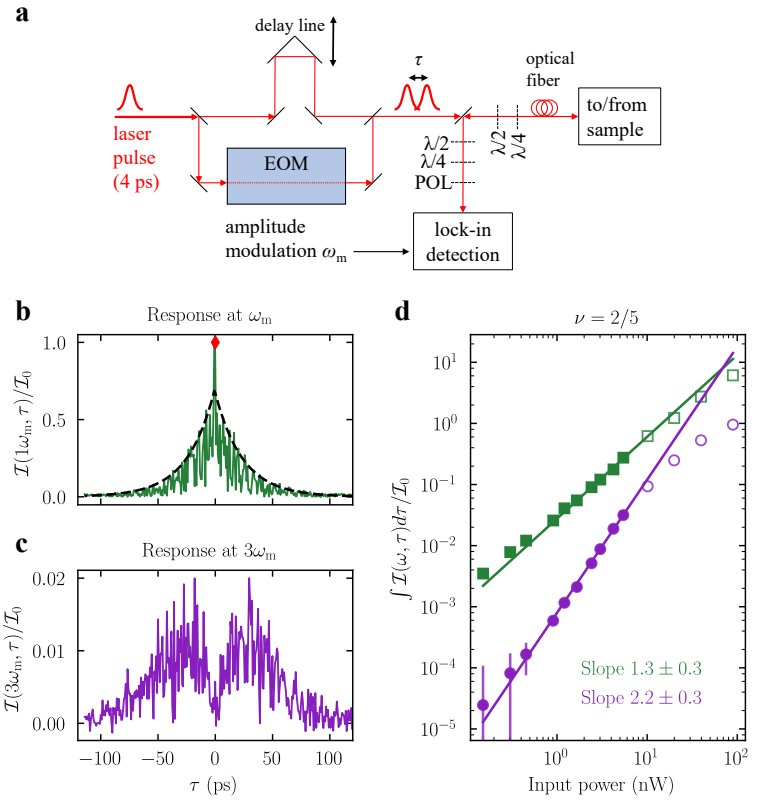

Figure 2: Time resolved measurement of interactions between polaron polaritons. a, Experimental setup. Two laser pulses separated by a variable delay $\tau$ generate an induced polarization in the sample. The emitted photons are sent onto an APD where linear contributions are separated from nonlinear contributions using an electro-optical modulator (EOM) to modulate one of the beams in amplitude. b. Typical linear and c, nonlinear interference signals obtained for $\nu=2 / 5(B=3.145 \mathrm{~T})$. All data is normalized to the maximal value of the linear response (red diamond). The dashed black line in $\mathbf{b}$ shows a double-sided exponential decay fitted to the envelope of the linear response to obtain $T_{\mathrm{LP}}$. The input average power was set to $I_{2}=2 \mathrm{nW}$. d, Evolution of the linear (green squares) and nonlinear (purple circles) signal integral values as a function of the incident optical power (double logarithmic plot). The input power is given as the average power of the delayed pulse (i.e. $I_{2}$ ), and the errorbars correspond to the statistical error on the counts only. We fit the data before saturation of the nonlinearity (full circles) by a power law (green and purple lines). Errors on the power law exponents are dominated by systematic errors on the input power.

sensitive to optical power due to possible photoionization of DX centers when illuminating the sample: increasing the optical power density may lead to unwanted modifications of $n_{e}$ and therefore to slow variations of the $(\nu$ dependent) polariton energies, which in turn may prevent us from properly evaluating the interactions. In order to isolate pure polariton-polariton interactions, we use a carefully designed sample structure with reduced sensitivity of $n_{e}$ to light, ${ }^{11}$ and perform time-resolved experiments in the pulsed-excitation regime in which the pulse duration $(\sim 4 \mathrm{ps})$ is shorter than the polariton lifetime $(>12 \mathrm{ps})$. A traditional approach to isolate the nonlinear contribution in four-wave mixing experiments consists in introducing an angle between the two exciting beams in order to generate a background-free nonlinear response at a different angle. ${ }^{35}$ However, the requirement for ultra-low temperatures render standard FWM experiments technically challenging to implement in our experimental geometry that uses a fiber coupled scanning confocal microscope in a dilution refrigerator. Note that access to the sample is restricted to reflection in colinear configuration: to separate the resonance fluorescence emitted by the sample from laser light that reflects off the surface, we use linearly polarized light in excitation and cross-polarized detection. In this way, we suppress the background laser light by 3-4 orders of magnitude, leaving the resonance fluorescence as the dominant contribution to the detected signal. We split the picosecond laser pulse into two paths, and introduce a variable delay $\tau$ before recombining them onto a beam splitter (see Extended Data Fig. 1). To distinguish the linear response from the (weaker) nonlinear response, we use an EOM placed between two crossed polarizers as an amplitude modulator in one arm of the interferometer. We apply a saw-tooth voltage profile to the EOM to create an electric field amplitude with sine modulation at frequency of $\omega_{\mathrm{m}} / 2 \pi=8011 \mathrm{~Hz}$. We optimize the EOM input voltage profile and the EOM alignment to realize a clean sine modulation at this frequency, with less than a percent of higher order harmonic contributions at $2,3,4 \times \omega_{\mathrm{m}}$. We finally couple the reflected signal to a single-mode fiber and send it to an APD, making sure that the count rate is well in the linear regime of the APD $\left(\sim 80000 \mathrm{~s}^{-1}\right)$. Due to the modulation, the power spectral density $\mathcal{I}(\omega, \tau)$ contains terms that oscillate at multiples of the modulation frequency $\omega_{\mathrm{m}}$. Expanding the first order terms in Equation (1)

$$
\left|P_{1}^{(1)}(t) \sin \left(\omega_{\mathrm{m}} t\right)\right|^{2}+\left|P_{2}^{(1)}(t, \tau)\right|^{2}+2 \Re\left(P_{1}^{(1)}(t)^{*} \sin \left(\omega_{\mathrm{m}} t\right) P_{2}^{(1)}(t, \tau)\right),
$$




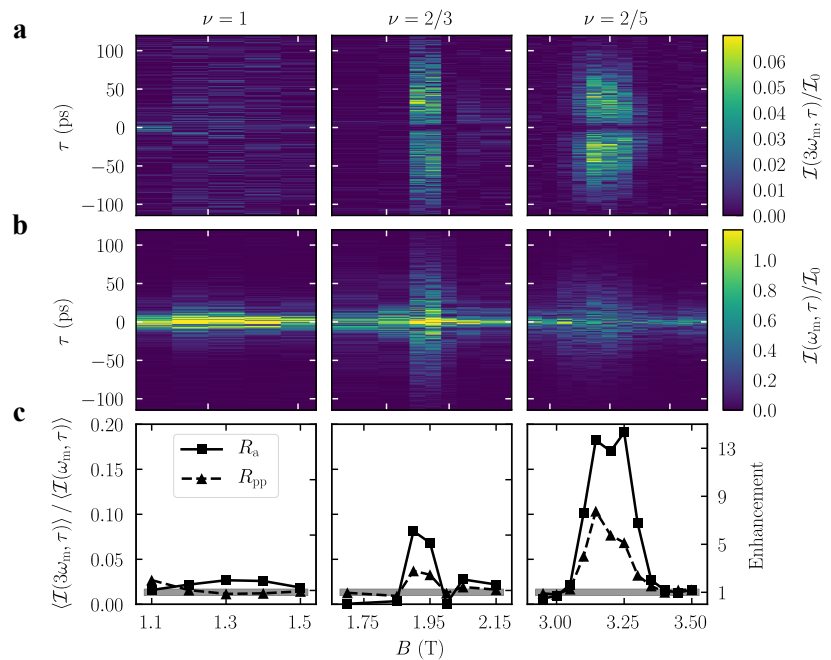

Figure 3: Enhancing interactions between quantum Hall polaritons at fractional filling factors. a, Nonlinear response $\mathcal{I}\left(3 \omega_{\mathrm{m}}, \tau\right) / \mathcal{I}_{0}$ and $\mathbf{b}$, linear response $\mathcal{I}\left(\omega_{\mathrm{m}}, \tau\right) / \mathcal{I}_{0}$ as a function of $B$, in vicinity of filling factors $\nu=1,2 / 3$ and 2/5. All data is normalized by the same value $\mathcal{I}_{0}$ as in Fig. $2 \mathrm{~b}$. c c, Enhancement of the nonlinearity, as revealed by the ratio of $3 \omega_{\mathrm{m}}$ and $\omega_{\mathrm{m}}$ responses. Two different measures of the enhancement strength are plotted, the area $R_{\mathrm{a}}$ integrated over $\tau$ (squares) and the signal peak-to-peak $R_{\mathrm{pp}}$ (triangles). In order to obtain a lower bound for the enhancement of interactions, we compare the signal to the noise level. Taking the outermost points in each panel as reference points, the $y$-axis on the right hand side gives the relative enhancement of $U$. The grey shaded area is the standard deviation of the reference points. The excitation power for all measurements is $I_{2}=20 \pm 3 \mathrm{nW}$.

we find that a field autocorrelation term appears at $\omega_{\mathrm{m}}$. The next order terms are the cross-products between linear and nonlinear polarizations with subscripts denoting fields originating from optical paths 1 and 2

$$
2 \Re\left(\left(P_{1}^{(1)^{*}} \sin \left(\omega_{\mathrm{m}} t\right)+P_{2}^{(1)^{*}}\right)\left(P_{111}^{(3)} \sin \left(\omega_{\mathrm{m}} t\right)^{3}+P_{112}^{(3)} \sin \left(\omega_{\mathrm{m}} t\right)^{2}+P_{122}^{(3)} \sin \left(\omega_{\mathrm{m}} t\right)+P_{222}^{(3)}\right)\right) .
$$

In turns out that $3 \omega_{\mathrm{m}}$ is the first frequency for which $P^{(3)}$ contributes to all terms with no background from $P^{(1)}$, so $\mathcal{I}\left(3 \omega_{\mathrm{m}}, \tau\right)$ is used to monitor the nonlinear response. Similar techniques have been used for four-wave mixing experiments in collinear geometry. ${ }^{36-41}$

The measurement procedure goes as follows. For a chosen magnetic field, we first set the cavity energy which defines the polaron content for $\mathrm{LP}_{\sigma^{-}}$. We then tune the laser pulse central energy to the $\mathrm{LP}_{\sigma^{-}}$resonance and suppress the reflected laser light. We note that when scanning $B$ (typically by few $100 \mathrm{mT}$ ) around a given filling factor (e.g. $\nu=2 / 5$ ) the singlet polaron resonance energy shift is small compared to the polariton normal mode splitting: ${ }^{42}$ as a consequence, we can keep the cavity energy constant while studying a given filling factor. We also keep the average intensities of pump (1) and probe (2) equal, which was found to result in a good signal to noise ratio. For each time delay $\tau$, we acquire photon counts for $1 \mathrm{~s}$, with the exception of Fig. 2 of the main text, where we used $10 \mathrm{~s}$ acquisition time. The APD sample frequency is $1 \mathrm{MHz}$, but data binning then leads to an effective sampling frequency of $9 \omega_{m}$. We then calculate the absolute value of the Fourier transform $I(t, \tau) \mapsto \mathcal{I}(\omega, \tau)$ for the recorded time traces, from which we extract frequency bins corresponding to the first multiples of $\omega_{\mathrm{m}}$. After background removal, we finally obtain $\mathcal{I}\left(\omega_{\mathrm{m}}, \tau\right)$ and $\mathcal{I}\left(3 \omega_{\mathrm{m}}, \tau\right)$ (where the background is derived by averaging $\mathcal{I}(\omega, \tau)$ in vicinity of the frequency of interest).

We perform a test experiment by red detuning the laser so our sample acts as a simple mirror. We adjust the detection polarizers such that the ADP count rate matches the one used in the main experiment. By applying the same experimental procedure to the signal, we observe that $\mathcal{I}\left(\omega_{\mathrm{m}}, \tau\right)$ corresponds to the laser pulse spectrum, whereas no signal is observed at the frequency $3 \omega_{\mathrm{m}}$. This excludes the possibility that the detector or any other optical elements in the setup contribute to the observed nonlinear signal. In another test experiment, we check the behaviour of $\mathcal{I}\left(3 \omega_{\mathrm{m}}, \tau\right)$ in response to cavity-polaron detuning. We observe that the nonlinear signal decreases when we red-detune the cavity with respect to the polaron energy: this is the expected behavior since the polaron content of the polaritons is decreased, and the polaritons are thus more photon-like.

Measurement and model of exciton-polariton interactions. We present, in this section, measurements on an undoped sample $\left(n_{e}=0\right)$, which does not contain a 2DES but only a neutral (intrinsic) QW. The goal is to measure the exciton-exciton interaction strength in a standard single QW (thickness $15 \mathrm{~nm}$ ) embedded in a DBR cavity and to compare it with known values of the interaction in order to establish our 
measurement technique as a viable tool for measuring interactions. In order to be able to have a single spin species and comparable conditions with the experimental work presented in the main text, namely linearly polarized excitation, orthogonal detection and $\sigma^{-}$polarized resonance, we performed this measurement under a $10 \mathrm{~T}$ magnetic field. ${ }^{43}$ To quantify the interaction, we compare our measurements with solutions of a single mode Gross-Pitaevskii equation, ${ }^{44,45}$ which requires that we estimate the polariton number $N=|\psi(t)|^{2}$ created by laser excitation in our experiment. Since we use pulsed resonant excitation with a low excitation duty cycle, we do not include in our model contributions from a dark exciton reservoir.

We estimate, in this paragraph, the polariton occupation number $N$ under picosecond laser pulsed excitation. The (Gaussian) laser pulse has a measured FWHM that is equal to $460 \mu \mathrm{eV}$, while the LP resonance (Lorentzian) showed a FWHM of $300 \mu \mathrm{eV}$. From this, we estimate the spectral overlap between the laser pulse and the LP line $\eta_{s}=0.57$. Based on white light reflectivity data (see Extended Data Fig. 2), we also estimate the exciton content $|X|^{2}=0.7$ and the coupling efficiency into the LP mode $\eta_{c}=0.24$ (see Lorentzian fits in Extended Data Fig. 2). Knowing the laser power impinging on the sample surface, we can estimate

$$
N=\eta_{s} \eta_{c} n_{\mathrm{ph}}
$$

where $n_{\mathrm{ph}}=p_{\mathrm{cw}} /\left(\hbar \omega_{\mathrm{L}} f_{\mathrm{rep}}\right)$ is the photon number per pulse, $p_{\mathrm{cw}}$ is the average input power, $f_{\mathrm{rep}}=76 \mathrm{MHz}$ is the pulse repetition rate and $\omega_{\mathrm{L}}$ is the laser center frequency.

To model the time-resolved experiment described above, we use a single mode Gross-Pitaevskii equation for the lower polariton wavefunction:

$$
\frac{d \psi(t)}{d t}=-\frac{\gamma}{2} \psi(t)-i g|\psi(t)|^{2} \psi(t)+F\left(t, \tau, t_{\mathrm{mod}}\right)
$$

where $g$ is the nonlinearity and $\gamma=0.1 \mathrm{ps}^{-1}$ is the cavity decay rate. The (modulated) drive term reads:

$$
F\left(t, \tau, t_{\mathrm{mod}}\right)=A_{1}\left(t_{\mathrm{mod}}\right) G(t)+A_{2} G(t-\tau) e^{i \omega_{\mathrm{L}} \tau}
$$

where $G(t)$ and $G(t-\tau)$ are 4 ps FWHM Gaussian envelopes delayed by $\tau, A_{1}$ is the (modulated) amplitude of the first pulse and $A_{2}$ the (constant) amplitude of the delayed pulse. In the simulation, we adjust the pulse amplitudes $A_{1}$ and $A_{2}$ to match the intracavity polariton number $N$ we estimated in the previous paragraph. The pulse intensities, averaged over a modulation cycle, are chosen to be equal.

We calculate $\psi$ for every $\tau$ and we repeat this procedure for different values of $A_{1}\left(t_{\mathrm{mod}}\right)=\sqrt{I_{1}} \sin \left(\omega_{\mathrm{m}} t_{\mathrm{mod}}\right)$, thus simulating the experimental procedure (time-resolved four-wave mixing). We then Fourier transform $\psi$ to obtain the calculated Fourier spectrum $\mathcal{I}_{\text {model }}(\omega, \tau)$ that we directly compare to the experiment as shown in Extended Data Fig. 3. In the end, the simulation includes only two free parameters: the interaction strength $g$ and a global scaling factor $\phi$ that accounts for the finite detection efficiency in our experiment $(\mathcal{I}(\omega, \tau)=$ $\phi \mathcal{I}_{\text {model }}(\omega, \tau)$ where $\phi$ is common to all values of $\omega$ and $\left.\tau\right)$. We determine the parameter $\phi$ by fitting $\mathcal{I}_{\text {model }}\left(\omega_{\mathrm{m}}, \tau\right)$ to our experiments. Then, we obtain $g$ by adjusting $\mathcal{I}_{\text {model }}\left(3 \omega_{m}, \tau\right)$ to best reproduce our measurements. Note that, given the estimate of $N=|\psi|^{2} \propto \mathcal{I}\left(\omega_{\mathrm{m}}, \tau\right)$, the information about $g$ is contained in the ratio of $\mathcal{I}\left(3 \omega_{\mathrm{m}}, \tau\right) / \mathcal{I}\left(1 \omega_{\mathrm{m}}, \tau\right)$, where $\phi$ drops out.

We show, in Extended Data Fig. 3, the results of our fit, which yields a value of $g=0.54 \pm 0.08 \mu \mathrm{eV}$ for the polariton interaction strength (accounting for statistical erros only, s.d.). To convert this single mode interaction energy into a $2 \mathrm{D}$ polariton-polariton interaction constant $U$, we multiply $g$ by the polariton mode area $A: U=A \times g$. Based on the numerical aperture of our objective N.A. $=0.15$, we expect the excitation beam to extend over $A=11 \mu \mathrm{m}^{2}$, which results in $U=6.2 \mu \mathrm{eV} \mu \mathrm{m}^{2}$. Finally, we estimate the exciton-exciton interaction strength by dividing $U$ by the exciton content squared:

$$
U_{X}=A \cdot g /|X|^{4}=12.5_{-2(9)}^{+2(18)} \mu \mathrm{eV} \mu \mathrm{m}^{2}
$$

This result is compatible with other values reported in the literature. ${ }^{4,5,14,18,31-33,46-50}$ The largest sources of errors on the measurement of $U_{X}$ originate from the estimate of $N$ and $A$ which are subject to large systematic errors (given in parentheses). Our estimate of $N$ could indeed easily be off by a factor of two. Additionally, our estimate of $A$ might deviate from the spot size estimate due to polariton diffusion and will eventually be modified by the exciton-exciton interactions. Altogether, this leads to the error estimate ${ }^{51}$ of Eq. 2. We observe, in Extended Data Fig. 3, a small deviation in the power dependence between experiment and fit. This discrepancy is due to a systematic calibration error of the input power (which also led to the observed deviation in the slopes measured in Fig. 2d of the main text). However, the resulting systematic error on $g$ is small compared to the first two contributions. We expect our measuring technique to give more accurate results in experimental geometries that allow imaging the polariton cloud in real-space and measuring in transmission.

Influence of the cavity detuning on the relative enhancement of $U$. We note that, since polaritons interact through their matter part, a change in the polaronic content of the polaritons as a function of $B$ 
would also lead to a modification of polariton-polariton interactions: polaritons with a higher polaron content will indeed show larger interactions. Therefore, for $\nu=2 / 5$, we may ask if part of the enhancement of the nonlinear signal, presented in Fig. 3 of the main text, could be due to variations in the matter content of LP as we vary the magnetic field. This is in fact not the case as we demonstrate below. As presented in the main text, our estimation of the enhancement of the nonlinearity is based on a comparison of the quantity $R_{\mathrm{a}}=\left\langle\mathcal{I}\left(3 \omega_{\mathrm{m}}, \tau\right)\right\rangle_{\tau} /\left\langle\mathcal{I}\left(\omega_{\mathrm{m}}, \tau\right)\right\rangle_{\tau}$ for different values of $\nu$. We note that $R_{a}$ is proportional to $U N$, where $N$ is the injected number of LP. For a pulsed excitation experiment where the pulse spectral width is larger than the polariton linewidth, $N$ is proportional to the LP photonic content $|C|^{2}=1-|X|^{2}$. Therefore, the nonlinear signal $R_{a}$ is proportional to $U N \propto|X|^{4}\left(1-|X|^{2}\right)$, which reaches its maximum for $|X|^{2}=2 / 3$. In the specific case of $\nu=2 / 5$, the lower polariton becomes more matter-like at $B=3.15 \mathrm{~T}\left(|X|^{2}=0.88\right)$ than it is at $B=3 \mathrm{~T}$ $\left(|X|^{2}=0.8\right)$. Because $|X|^{4}\left(1-|X|^{2}\right)$ is a decreasing function of $|X|^{2}$ for $|X|^{2}>2 / 3$, it turns out the observed change in matter component by itself cannot explain the measured increase of $R_{a}$. As a result, we conclude that our estimation of the enhancement of $U$ based on the evolution of $R_{a}$ around $\nu=2 / 5$ is conservative and that we may be underestimating the increase of $U$ by not taking into account the changes in $|X|^{2}$.

Interaction strength in fractional quantum Hall states. We have demonstrated, in Fig. 3 of the main text, that the nonlinear response of polaron polaritons increases as we tune the system to fractional quantum Hall filling factors. This enabled us to quantify the enhancement of polariton interactions at fractional filling factors as compared to more generic filling factors in the vicinity of these particular fractional quantum Hall states. Comparing the interaction strength between fractional quantum Hall polaritons to the more general case of an undoped sample thus calls for an absolute measurement of the interaction strength. To do so, we apply the procedure detailed above to quantum Hall polaritons at 2DES filling factors $\nu=2 / 5$ and $\nu=2 / 3$. As pointed out in the main text, some features observed in the time evolution of $\mathcal{I}\left(3 \omega_{\mathrm{m}}, \tau\right)$ for fractional quantum Hall polaritons are not captured by our mean-field model. Most notably, although the mean-field model predicts that $\mathcal{I}\left(3 \omega_{\mathrm{m}}, \tau\right)$ only takes large values for positive delays (see $\mathcal{I}\left(3 \omega_{\mathrm{m}}, \tau\right)$ in Extended Data Fig. 3), we observe, in the fractional quantum Hall polariton case, that $\mathcal{I}\left(3 \omega_{\mathrm{m}}, \tau\right)$ is symmetric about $\tau=0$ and thus takes large values for negative delays as well (see for example the symmetric shape of $\mathcal{I}\left(3 \omega_{\mathrm{m}}, \tau\right)$ in Fig. 2 of the main text). To obtain a quantitative estimate of the interaction constant of fractional quantum Hall polaritons that we can compare to the value obtained for exciton polaritons in a neutral quantum well, we therefore apply the procedure described in the previous paragraph (measurement and model of exciton-polariton interactions), where we restrict the fit to the signal observed at positive delays.

We summarize, in Extended Data Fig. 4, the fitting procedure for the $\nu=2 / 5$ case. We fit the white light reflectivity spectrum obtained at $B=3.2 \mathrm{~T}$ by a sum of three Lorentzian functions to extract the LP linewidth $\gamma=67 \pm 5 \mu \mathrm{eV}$, the LP cavity content $\simeq 0.12$ and LP peak amplitude $\simeq 0.40$. We note that the LP linewidth is narrower than the bare cavity linewidth $\left(\gamma_{\text {cav }}=275 \mu \mathrm{eV}\right)$ despite the large matter component of $88 \%$ : reduction of the cavity content has resulted in a reduction of the polariton linewidth as would be expected in a sample where excitons only decay through their coupling to the cavity. This indicates that the presence of the 2DES does not broaden the lower polariton linewidth. We then repeat the steps described in above to estimate the interaction constant. The lifetime was adjusted to $\gamma=16 \mathrm{ps}$ to account for the observed narrowing of the linewidth. At $\nu=2 / 5$, we obtain $U=57_{-7(40)}^{+7(80)} \mu \mathrm{eV} \mu \mathrm{m}^{2}$. As discussed before, the large systematic errors (in parentheses) stem from the uncertainty of the polariton number estimate and mode area, which are kept the same for the undoped QW sample as well as for the 2DES sample measurements. For estimating the relative enhancement of interactions, note that the only relevant error bar is statistical $\left(U=57 \pm 7 \mu \mathrm{eV} \mu \mathrm{m}^{2}\right)$ since the systematic error affects all the measurements the same way.

The results for of our measurements of $U$ are summarized in Extended Data Tab. 1 for the 2DEG sample at different filling factors as well as for the undoped sample. In the table, we only display the statistical error bars that are relevant to compare relative variations of $U$ between columns of the Table. At $\nu=2 / 5$, we observe that interactions are enhanced by more than an order of magnitude as compared to a neighboring filling factor (taken at $B=3.0 \mathrm{~T}$ ). This is consistent with our estimation of the enhancement of interaction based on measuring the area of $\mathcal{I}\left(3 \omega_{\mathrm{m}}, \tau\right)$. Compared to the undoped QW, we find that $U$ is increased by a factor $4.6 \pm 0.9$ at $\nu=2 / 5$. This result indicates that away from $\nu=2 / 5$ and $\nu=2 / 3$, polaron-polariton interactions may be reduced as compared to exciton-polaritons in the neutral quantum well. Nevertheless, in the $\nu=2 / 5$ case, the ratio $U /(A \gamma)$ approaches unity (for $A=1 \mu \mathrm{m}^{2}$ ), which indicates that polariton blockade is within reach.

Increase in polariton coherence time. We have observed, in Fig. 3b of the main text, an interesting evolution of the (mostly linear) term $\mathcal{I}\left(\omega_{\mathrm{m}}, \tau\right)$ as we tune $B$ : the characteristic decay time of $\mathcal{I}\left(\omega_{\mathrm{m}}, \tau\right)($ i.e. the polariton coherence time $\left.T_{\mathrm{LP}}\right)$ increases for $\nu=2 / 3$ and $\nu=2 / 5$. We present, in this section, a detailed study of this effect versus input pump power. We show, in Extended Data Fig. 5a, a semilog plot of a typical trace $\mathcal{I}\left(\omega_{\mathrm{m}}, \tau\right)$ (blue). To extract $T_{\mathrm{LP}}$, we fit the envelope of this trace by a double exponential decay (black line): $T_{\mathrm{LP}}$ is directly given by the exponential decay time. In Extended Data Fig. 5b-d, we plot the fitted values of 
$T_{\mathrm{LP}}$ as a function of input pump power around filling factors $\nu=1,2 / 3,2 / 5$. In every panel, we show a dataset recorded when $B$ is tuned to the corresponding quantum Hall states (blue points), and another dataset recorded at a slightly different filling factor (orange points). At exactly $\nu=2 / 3$ and $\nu=2 / 5$, we observe that $T_{\mathrm{LP}}$ first increases sharply and then stabilizes at a value two to three times larger to its low-power value. This increase of $T_{\mathrm{LP}}$ coincides with the enhancement (and high power saturation) of the nonlinearity at filling factors $\nu=2 / 3$ and $\nu=2 / 5$ discussed in the main text. In stark contrast, slightly away from these filling factors, as well as for $\nu=1, T_{\mathrm{LP}}$ stays relatively stable around its low power value. In another set of experiments, we extracted $T_{\mathrm{LP}}$ by measuring the Lorentzian width of $L P_{\sigma-}$ in white light reflectivity spectra as a function of input power; this study (not shown here) led to the same observations. These results suggest a nonlinear behavior of $\mathcal{I}\left(\omega_{\mathrm{m}}, \tau\right)$ at fractional filling factors. However, monitoring the average value $\left\langle\mathcal{I}\left(\omega_{\mathrm{m}}, \tau\right)\right\rangle_{\tau}$ versus power (see Fig.2d of the main text) shows that $\left\langle\mathcal{I}\left(\omega_{\mathrm{m}}, \tau\right)\right\rangle_{\tau}$ remains linear in excitation power. At this stage, the origin of this power dependent enhancement of $T_{\mathrm{LP}}$ thus remains unknown. While the measured increase in nonlinearity is clearly an advantage for implementing strongly interacting polaritons, it is unclear whether the observed (high power) increased coherence time (and thus decreased linewidth) could also be beneficial for realizing polariton blockade.

Repeating the measurements for a higher density sample. We repeat our measurement using another sample with higher density electron density of the 2DES $\left(n_{e}=1.4 \times 10^{11} \mathrm{~cm}^{-2}\right)$. We plot, in Extended Data Fig. 6a, the white light reflectivity measurement recorded in $\sigma^{-}$polarization around filling factor $\nu=2 / 3$ $(B \simeq 8.6 \mathrm{~T})$. Note that, contrary to the lower electron density sample presented in Fig. $1 \mathrm{~b}$ of the main text, the $\nu=2 / 3$ state is spin-polarized at this magnetic field. This is observed in the reflection spectrum of Extended Data Fig. 6a, that resembles the spectrum recorded for the (spin-polarized) state at $\nu=2 / 5$ in the low density sample sample (see the sharp reduction of normal mode splitting at $B=8.65 \mathrm{~T}$ ). We note however that the coupling efficiency of incident light into the polariton modes was reduced in the high-density sample. We show, in Fig S6b, the results of our time-resolved four-wave mixing measurement around $\nu=2 / 3$. We observe a clear nonlinear response $\mathcal{I}\left(3 \omega_{\mathrm{m}}, \tau\right)$ when $\nu$ is tuned to $2 / 3$ exactly. As we go away from $\nu=2 / 3$, the nonlinearity decreases (bottom row). This behavior is very similar to the one presented in the main text for the low-density sample, since we observe a strong dependence on the filling factor of the nonlinear response $\mathcal{I}\left(3 \omega_{\mathrm{m}}, \tau\right)$. The top row also shows the linear response $\mathcal{I}\left(\omega_{\mathrm{m}}, \tau\right)$ for comparison, where we observe the increase of $T_{\mathrm{LP}}$ for $\nu=2 / 3$ (see variations in the exponential decay time of $\left.\mathcal{I}\left(\omega_{\mathrm{m}}, \tau\right)\right)$. With this measurement, we demonstrate the repeatability of our measurement, using another sample with higher electron density. A quantitative comparison of the interaction strengths between the two samples, is however rendered difficult due to the different experimental conditions relevant for the two samples; in particular, due to the strong difference in coupling efficiency of incident light into the polariton modes in the two samples.

\section{References}

[34] Stepanov, P. et al. Two-components nature of the excitations in a polariton superfluid. arXiv:1810.125\%0 [cond-mat] (2018). URL http://arxiv.org/abs/1810.12570. ArXiv: 1810.12570.

[35] Boyd, R. W. Nonlinear Optics (Elsevier, 2008).

[36] Hall, K. L., Lenz, G., Ippen, E. P. \& Raybon, G. Heterodyne pump-probe technique for time-domain studies of optical nonlinearities in waveguides. Optics Letters 17, 874-876 (1992). URL https://www. osapublishing.org/ol/abstract. cfm?uri=ol-17-12-874.

[37] Mecozzi, A. \& Mørk, J. Transient and time-resolved four-wave mixing with collinear pump and probe pulses using the heterodyne technique. Pure and Applied Optics: Journal of the European Optical Society Part A 7, 335 (1998). URL http://stacks.iop.org/0963-9659/7/i=2/a=023.

[38] Patton, B., Woggon, U. \& Langbein, W. Coherent Control and Polarization Readout of Individual Excitonic States. Physical Review Letters 95 (2005). URL https ://link. aps .org/doi/10.1103/PhysRevLett. 95. 266401.

[39] Kohnle, V. et al. Four-wave mixing excitations in a dissipative polariton quantum fluid. Physical Review B 86 (2012). URL https://link.aps.org/doi/10.1103/PhysRevB.86.064508.

[40] Nardin, G., Autry, T. M., Silverman, K. L. \& Cundiff, S. T. Multidimensional coherent photocurrent spectroscopy of a semiconductor nanostructure. Optics Express 21, 28617 (2013). URL https://www . osapublishing.org/oe/abstract. cfm?uri=oe-21-23-28617.

[41] Smallwood, C. L. \& Cundiff, S. T. Multidimensional Coherent Spectroscopy of Semiconductors. Laser \& Photonics Reviews 12, 1800171 (2018). URL https://onlinelibrary.wiley.com/doi/full/10.1002/ Ipor. 201800171. 
[42] This does not imply a small energy shift for the polariton formed from this singlet polaron, as presented in Fig. 1c of the main text.

[43] For $B=10 \mathrm{~T}$, we do not expect the magnetic field to strongly influence the strength of exciton-exciton interactions since the exciton Bohr radius is still of the same order as the magnetic length.

[44] Wouters, M. \& Carusotto, I. Excitations in a Nonequilibrium Bose-Einstein Condensate of Exciton Polaritons. Physical Review Letters 99 (2007). URL https://link.aps.org/doi/10.1103/PhysRevLett.99. 140402.

[45] Keeling, J. \& Berloff, N. G. Spontaneous Rotating Vortex Lattices in a Pumped Decaying Condensate. Physical Review Letters 100 (2008). URL https://link.aps.org/doi/10.1103/PhysRevLett. 100. 250401 .

[46] Ciuti, C., Savona, V., Piermarocchi, C., Quattropani, A. \& Schwendimann, P. Role of the exchange of carriers in elastic exciton-exciton scattering in quantum wells. Physical Review B 58, 7926-7933 (1998). URL https://link.aps.org/doi/10.1103/PhysRevB.58.7926.

[47] Tassone, F. \& Yamamoto, Y. Exciton-exciton scattering dynamics in a semiconductor microcavity and stimulated scattering into polaritons. Physical Review B 59, 10830-10842 (1999). URL https://link. aps.org/doi/10.1103/PhysRevB.59.10830.

[48] Amo, A. et al. Superfluidity of polaritons in semiconductor microcavities. Nature Physics 5, 805-810 (2009). URL https://www. nature.com/articles/nphys1364.

[49] Byrnes, T., Kolmakov, G. V., Kezerashvili, R. Y. \& Yamamoto, Y. Effective interaction and condensation of dipolaritons in coupled quantum wells. Physical Review B 90 (2014). URL https://link.aps.org/ doi/10.1103/PhysRevB.90.125314.

[50] Nalitov, A. V., Solnyshkov, D. D., Gippius, N. A. \& Malpuech, G. Voltage control of the spin-dependent interaction constants of dipolaritons and its application to optical parametric oscillators. Physical Review $B 90$ (2014). URL https://link.aps.org/doi/10.1103/PhysRevB.90.235304.

[51] Note that $U_{X}$ is inversely proportional to the estimated polariton number $N$.

Data availability. The data that support the findings of this study are available at the ETH Research Collection (URL http://hdl.handle.net/20.500.11850/338463) DOI:10.3929/ethz-b-000338463 
Extended Data Fig. 1: Experimental setup. Schematic of the interferometer used for measuring the nonlinear response of the system.

Extended Data Fig. 2: White light reflectivity measurements. a, Evolution of the reflectivity spectra as we tune the cavity energy across the exciton resonance. The red line marks the cavity energy for the spectrum shown in the right panel. b, Background subtracted spectrum (blue dots). The black line shows Lorentzian fits to the spectrum. From the peak areas, we determine the exciton content $|X|^{2}=0.7$. The lower polariton amplitude is $\eta_{c}=0.24$.

Extended Data Fig. 3: Comparing data from undoped QW sample to GPE. Top row: comparison between the measured (green circles) and calculated (red shaded area) $\mathcal{I}\left(\omega_{\mathrm{m}}, \tau\right)$ for different input powers, used to calibrate the detection efficiency $\phi$. Bottom row: comparison between the measured (purple circles) and calculated (red shaded area) $\mathcal{I}\left(3 \omega_{\mathrm{m}}, \tau\right)$ for different input powers, yields a value of $g=0.54 \mu \mathrm{eV}$ for the polariton interaction strength.

Extended Data Fig. 4: Esitmation of interaction constant at 2/5. a, White light reflectivity spectra as a function of magnetic field. $\mathbf{b}$, Linecut of the data (blue circles) at $B=3.2 \mathrm{~T}$ with a fit (black line) consisting of three Lorentzian resonances. c, Comparison of the linear $\mathcal{I}\left(\omega_{\mathrm{m}}, \tau\right)$ (top row, green circles) and nonlinear response $\mathcal{I}\left(3 \omega_{\mathrm{m}}, \tau\right)$ (bottom row, purple circles) at $\nu=2 / 5$ with the GPE model (red).

Extended Data Tab. 1: Comparison of interaction constants and LP linewidths. Table summarizing the estimated interaction constants and fitted linewidths for the undoped and 2DES samples. The polariton number $N$ is estimated as described in the Methods (Measurement and model of exciton-polariton interactions) and given per cw-equivalent laser excitation power. The quoted error bars are statistical (s.d.).

Extended Data Fig. 5: Increase in polariton coherence time with input power at fractional quantum Hall states. a, Extraction of $T_{\mathrm{LP}}$, showing an exemplary linear response in a logarithmic plot with the fit to the envelope in green. The inverse slope corresponds to $T_{\mathrm{LP}}$. a-d, Dependence of $T_{\mathrm{LP}}$ on input power for the filling factors considered in the main text. Blue circles correspond to the magnetic field at the quantum Hall state, orange circles to a magnetic field nearby.

Extended Data Fig. 6: Additional data from another sample. a, White light reflectivity spectrum recorded using $\sigma^{-}$polarized light. At $B=8.6 \mathrm{~T}$, the optical signature of $\nu=2 / 3$ shows as a reduction of the polariton splitting around $1527 \mathrm{meV}$ (note that the upper polariton is particularly faint). $\mathbf{b}, \mathrm{FWM}$ experiment around filling factor $\nu=2 / 3$. The top row shows $\mathcal{I}\left(\omega_{\mathrm{m}}, \tau\right)$ while the bottom row shows $\mathcal{I}\left(3 \omega_{\mathrm{m}}, \tau\right)$. All data has been normalized to the maximal value of $\mathcal{I}\left(\omega_{\mathrm{m}}, \tau\right)$ at $B=8.65 \mathrm{~T}$ (red diamond). The integration time was chosen equal to $10 \mathrm{~s}$ and the input power was $35 \pm 5 \mathrm{nW}$. 